\title{
On monotone Ćirić quasi-contraction mappings with a graph
}

\section{Monther Rashed Alfuraidan*}

"Correspondence: monther@kfupm.edu.sa

Department of Mathematics and Statistics, King Fahd University of Petroleum and Minerals, Dhahran, 31261, Saudi Arabia

\begin{abstract}
In this paper, we obtain sufficient conditions for the existence of fixed points for monotone quasi-contraction mappings in metric and modular metric spaces endowed with a graph. This is the extension of Ran and Reurings and Jachymski fixed point theorems for monotone contraction mappings in partially ordered metric spaces and in metric spaces endowed with a graph to the case of quasi-contraction mappings introduced by Ćirić.
\end{abstract}

MSC: Primary 47H09; secondary 47H10

Keywords: fixed point; modular metric space; monotone mappings; quasi-contraction; directed graph

\section{Introduction}

Banach's contraction principle [1] is remarkable in its simplicity, yet it is perhaps the most widely applied fixed point theorem in all of analysis. This is because the contractive condition on the mapping is simple and easy to test, it requires only a complete metric space for its setting, and it finds almost canonical applications in the theory of differential and integral equations. Over the years, many mathematicians tried successfully to extend this fundamental theorem. Recently a version of this theorem was given in partially ordered metric spaces [2, 3] and in metric spaces with a graph [4]. In this work, we discuss the case of quasi-contractive mappings defined in partially ordered metric spaces and modular metric spaces endowed with a graph.

For more on metric fixed point theory, the reader may consult the book [5].

\section{Graph basic definitions}

The terminology of graph theory instead of partial ordering gives a wider picture and yield interesting generalization of the Banach contraction principle. In this section, we give the basic graph theory definitions and notations which will be used throughout.

A graph is an ordered pair $(V, E)$ where $V$ is a set and $E$ is a binary relation on $V(E \subseteq V \times$ $V)$. Elements of $E$ are called edges. We are concerned here with directed graphs (digraphs) that have a loop at every vertex (i.e., $(a, a) \in E$ for each $a \in V)$. Such digraphs are called reflexive. In this case $E \subseteq V \times V$ corresponds to a reflexive (and symmetric) binary relation on $V$. Moreover, we may treat $G$ as a weighted graph by assigning to each edge the distance between its vertices. By $G^{-1}$ we denote the conversion of a graph $G$, i.e., the graph obtained

(c) 2015 Alfuraidan. This article is distributed under the terms of the Creative Commons Attribution 4.0 International License (http://creativecommons.org/licenses/by/4.0/), which permits unrestricted use, distribution, and reproduction in any medium, provided you give appropriate credit to the original author(s) and the source, provide a link to the Creative Commons license, and indicate if changes were made. 
from $G$ by reversing the direction of edges. Thus we have

$$
E\left(G^{-1}\right)=\{(y, x) \mid(x, y) \in E(G)\} .
$$

A digraph $G$ is called an oriented graph if whenever $(u, v) \in E(G)$, then $(v, u) \notin E(G)$. The letter $\widetilde{G}$ denotes the undirected graph obtained from $G$ by ignoring the direction of edges. Actually, it will be more convenient for us to treat $\widetilde{G}$ as a directed graph for which the set of its edges is symmetric. Under this convention,

$$
E(\widetilde{G})=E(G) \cup E\left(G^{-1}\right) .
$$

Given a digraph $G=(V, E)$, a (di)path of $G$ is a sequence $a_{0}, a_{1}, \ldots, a_{n}, \ldots$ with $\left(a_{i}, a_{i+1}\right) \in$ $E(G)$ for each $i=0,1,2, \ldots$. A finite path $\left(a_{0}, a_{1}, \ldots, a_{n}\right)$ is said to have length $n+1$ for $n \in \mathbb{N}$. A closed directed path of length $n>1$ from $x$ to $y$, i.e., $x=y$, is called a directed cycle. An acyclic digraph is a digraph that has no directed cycle. A digraph is connected if there is a finite (di)path joining any two of its vertices and it is weakly connected if $\widetilde{G}$ is connected.

Definition 2.1 A digraph $G$ is transitive if

$$
(x, y) \in E(G) \quad \text { and } \quad(y, z) \in E(G) \quad \Rightarrow \quad(x, z) \in E(G) \quad \text { for all } x, y, z \in V(G) .
$$

As Jachymski [4] did, we introduce the following property.

Let $(X, d)$ be a metric space and $G$ be a reflexive digraph defined on $X$. We say that $E(G)$ has property $(*)$ if

(*) For any $\left(x_{n}\right)_{n \geq 1}$ in $X$, if $x_{n} \rightarrow x$ and $\left(x_{n}, x_{n+1}\right) \in E(G)$ for $n \geq 1$, then there is a subsequence $\left(x_{k_{n}}\right)_{n \geq 1}$ with $\left(x_{k_{n}}, x\right) \in E(G)$ for $n \geq 1$.

Note that if $G$ is a reflexive transitive digraph defined on $X$, then property $(*)$ implies the following property:

(**) For any $\left(x_{n}\right)_{n \geq 1}$ in $X$, if $x_{n} \rightarrow x$ and $\left(x_{n}, x_{n+1}\right) \in E(G)$ for $n \geq 1$, then $\left(x_{n}, x\right) \in E(G)$ for every $n \geq 1$.

Let us finish this section with the following example of a transitive cyclic digraph which cannot be a partial order. Therefore our approach is different from the one used in [6] which is based on the use of a partial order in Banach and metric spaces.

Example 2.1 Let $\left(\mathbb{R}^{2}, d\right)$ be the Euclidean plane. Define the digraph $G$ on $\mathbb{R}^{2}$ by: $(x, y) \in E(G)$ if and only if $x_{2} \leq y_{2}$, where $x=\left(x_{1}, x_{2}\right)$ and $y=\left(y_{1}, y_{2}\right)$ are in $\mathbb{R}^{2}$. Then $G$ is reflexive, transitive for which $G$-intervals are closed. Note that $G$ contains cycles. Indeed, we have $(x, y) \in E(G)$ and $(y, x) \in E(G)$, where

$$
x=(1,0) \text { and } y=(2,0) \text {. }
$$

Therefore the graph $G$ will not be generated by a partial order.

For more examples on the use of graph theory with fixed point theory, the reader may see [7]. 


\section{G-Monotone quasi-contraction mappings in metric spaces}

As a generalization to the Banach contraction principle, Ćirić [8] introduced the concept of quasi-contraction mappings. In this section, we investigate monotone mappings which are quasi-contraction mappings. Throughout this section we assume that $(X, d)$ is a metric space and $G$ is a reflexive transitive digraph defined on $X$. Moreover, we assume that $E(G)$ has property $(*)$ and $G$-intervals are closed. Recall that a $G$-interval is any of the subsets $[a, \rightarrow)=\{x \in C ;(a, x) \in E(G)\}$ and $(\leftarrow, b]=\{x \in C ;(x, b) \in E(G)\}$ for any $a, b \in C$.

Definition 3.1 Let $C$ be a nonempty subset of $X$. A mapping $T: C \rightarrow C$ is called:

(1) $G$-monotone if $T$ is edge preserving, i.e., $(T(x), T(y)) \in E(G)$ whenever $(x, y) \in E(G)$ for any $x, y \in C$.

(2) $G$-monotone quasi-contraction if $T$ is $G$-monotone and there exists $k<1$ such that for any $x, y \in C,(x, y) \in E(G)$, we have

$$
d(T(x), T(y)) \leq k \max (d(x, y) ; d(x, T(x)) ; d(y, T(y)) ; d(x, T(y)) ; d(y, T(x))) .
$$

The point $x \in C$ is called a fixed point of $T$ if $T(x)=x$. The set of fixed points of $T$ will be denoted by $\operatorname{Fix}(T)$.

In the sequel we prove an existence fixed point theorem for such mappings. First, let $T$ and $C$ be as in Definition 3.1. For any $x \in C$, define the orbit $\mathcal{O}(x)=\left\{x, T(x), T^{2}(x), \ldots\right\}$, and its diameter by

$$
\delta(x)=\sup \left\{d\left(T^{n}(x), T^{m}(x)\right): n, m \in \mathbb{N}\right\} .
$$

The following technical lemma is crucial to prove the main result of this section.

Lemma 3.1 Let $(X, d)$ and $G$ be as above. Let $C$ be a nonempty subset of $X$ and $T: C \rightarrow C$ be a G-monotone quasi-contraction mapping. Let $x \in C$ be such that $(x, T(x)) \in E(G)$ and $\delta(x)<\infty$. Then, for any $n \geq 1$, we have

$$
\delta\left(T^{n}(x)\right) \leq k^{n} \delta(x)
$$

where $k<1$ is the constant associated with the G-monotone quasi-contraction definition of T. Moreover, we have

$$
d\left(T^{n}(x), T^{n+m}(x)\right) \leq k^{n} \delta(x)
$$

for any $n, m \in \mathbb{N}$.

Proof Since $T$ is $G$-monotone, then $\left(T^{n}(x), T^{n+1}(x)\right) \in E(G)$ for any $n \in \mathbb{N}$. By transitivity of the graph $G$, we have $\left(T^{n}(x), T^{m}(x)\right) \in E(G)$ for any $n, m \in \mathbb{N}$. Hence

$$
\begin{aligned}
d\left(T^{n}(x), T^{m}(x)\right) \leq & k \max \left(d\left(T^{n-1}(x), T^{m-1}(x)\right) ; d\left(T^{n-1}(x), T^{n}(x)\right) ;\right. \\
& \left.d\left(T^{m-1}(x), T^{m}(x)\right) ; d\left(T^{n-1}(x), T^{m}(x)\right) ; d\left(T^{n}(x), T^{m-1}(x)\right)\right)
\end{aligned}
$$


for any $n, m \geq 1$. This obviously implies that

$$
\delta\left(T^{n}(x)\right) \leq k \delta\left(T^{n-1}(x)\right), \quad n \geq 1 .
$$

Hence

$$
\delta\left(T^{n}(x)\right) \leq k^{n} \delta(x), \quad n \geq 1
$$

This will imply

$$
d\left(T^{n}(x), T^{n+m}(x)\right) \leq \delta\left(T^{n}(x)\right) \leq k^{n} \delta(x)
$$

for any $n, m \in \mathbb{N}$.

Using Lemma 3.1, we prove the main result of this section.

Theorem 3.1 Let $(X, d)$ and $G$ be as above. Assume that $(X, d)$ is complete. Let $C$ be a closed nonempty subset of $X$ and $T: C \rightarrow C$ be a G-monotone quasi-contraction mapping. Let $x \in C$ be such that $(x, T(x)) \in E(G)$ and $\delta(x)<\infty$. Then:

(i) $\left\{T^{n}(x)\right\}$ converges to $\omega \in C$ which is a fixed point of $T$ and $(x, \omega) \in E(G)$. Moreover, we have

$$
d\left(T^{n}(x), \omega\right) \leq k^{n} \delta(x), \quad n \geq 1 .
$$

(ii) If $z$ is a fixed point of $T$ such that $(x, z) \in E(G)$, then $z=\omega$.

Proof Let us prove (i). Lemma 3.1 implies that $\left\{T^{n}(x)\right\}$ is Cauchy. Since $X$ is complete and $C$ is closed, then there exists $\omega \in C$ such that $\left\{T^{n}(x)\right\}$ converges to $\omega$. Since

$$
d\left(T^{n}(x), T^{n+m}(x)\right) \leq k^{n} \delta(x), \quad n, m \in \mathbb{N},
$$

we let $m \rightarrow \infty$ to get

$$
d\left(T^{n}(x), \omega\right) \leq k^{n} \delta(x), \quad n \geq 1 .
$$

Since $T$ is $G$-monotone, we get $\left(T^{n}(x), T^{n+1}(x)\right) \in E(G)$ for any $n \geq 1$. By property $(* *)$, we conclude that $\left(T^{n}(x), \omega\right) \in E(G)$ for any $n \geq 1$. In particular, we have $(x, \omega) \in E(G)$. In order to show that $\omega$ is a fixed point of $T$, note that we have

$$
\begin{aligned}
d\left(T^{n}(x), T(\omega)\right) \leq & k \max \left(d\left(T^{n-1}(x), \omega\right) ; d\left(T^{n-1}(x), T^{n}(x)\right) ;\right. \\
& \left.d(\omega, T(\omega)) ; d\left(T^{n-1}(x), T(\omega)\right) ; d\left(T^{n}(x), \omega\right)\right)
\end{aligned}
$$

for any $n \geq 1$. If we let $n \rightarrow+\infty$, we get $d(\omega, T(\omega)) \leq k d(\omega, T(\omega))$, which forces $d(\omega, T(\omega))=$ 0 since $k<1$. Therefore we have $T(\omega)=\omega$.

Next we show (ii). Let $z \in C$ be a fixed point of $T$ such that $(x, z) \in E(G)$. Then we have

$$
d\left(T^{n}(x), z\right) \leq k \max \left(d\left(T^{n-1}(x), z\right) ; d\left(T^{n-1}(x), T^{n}(x)\right) ; d\left(T^{n}(x), z\right)\right)
$$


for any $n \geq 2$. If we let $n \rightarrow+\infty$, we get

$$
d(\omega, z)=\limsup _{n \rightarrow \infty} d\left(T^{n}(x), z\right) \leq k \limsup _{n \rightarrow \infty} d\left(T^{n}(x), z\right)=k d(\omega, z) .
$$

Since $k<1$, we get $d(\omega, z)=0$, i.e., $\omega=z$.

In the next section, we discuss the validity of Theorem 3.1 in modular metric spaces. This is a very important class of spaces since they are similar to metric spaces in their structure but without the triangle inequality and offer a wide range of applications.

\section{G-Monotone quasi-contraction mappings in modular metric spaces}

Let $X$ be a nonempty set. Throughout this section for a function $\omega:(0, \infty) \times X \times X \rightarrow$ $(0, \infty)$, we will write

$$
\omega_{\lambda}(x, y)=\omega(\lambda, x, y)
$$

for all $\lambda>0$ and $x, y \in X$.

Definition 4.1 $[9,10]$ A function $\omega:(0, \infty) \times X \times X \rightarrow[0, \infty]$ is said to be a modular metric on $X$ if it satisfies the following axioms:

(i) $x=y$ if and only if $\omega_{\lambda}(x, y)=0$ for all $\lambda>0$;

(ii) $\omega_{\lambda}(x, y)=\omega_{\lambda}(y, x)$ for all $\lambda>0$ and $x, y \in X$;

(iii) $\omega_{\lambda+\mu}(x, y) \leq \omega_{\lambda}(x, z)+\omega_{\mu}(z, y)$ for all $\lambda, \mu>0$ and $x, y, z \in X$.

If instead of (i) we have only the condition (i')

$$
\omega_{\lambda}(x, x)=0 \quad \text { for all } \lambda>0, x \in X
$$

then $\omega$ is said to be a pseudomodular (metric) on $X$. A modular metric $\omega$ on $X$ is said to be regular if the following weaker version of (i) is satisfied

$$
x=y \quad \text { if and only if } \quad \omega_{\lambda}(x, y)=0 \quad \text { for some } \lambda>0 \text {. }
$$

Finally, $\omega$ is said to be convex if for $\lambda, \mu>0$ and $x, y, z \in X$, it satisfies the inequality

$$
\omega_{\lambda+\mu}(x, y) \leq \frac{\lambda}{\lambda+\mu} \omega_{\lambda}(x, z)+\frac{\mu}{\lambda+\mu} \omega_{\mu}(z, y)
$$

Note that for a metric pseudomodular $\omega$ on a set $X$, and any $x, y \in X$, the function $\lambda \rightarrow$ $\omega_{\lambda}(x, y)$ is nonincreasing on $(0, \infty)$. Indeed, if $0<\mu<\lambda$, then

$$
\omega_{\lambda}(x, y) \leq \omega_{\lambda-\mu}(x, x)+\omega_{\mu}(x, y)=\omega_{\mu}(x, y)
$$

Definition 4.2 $[9,10]$ Let $\omega$ be a pseudomodular on $X$. Fix $x_{0} \in X$. The two sets

$$
X_{\omega}=X_{\omega}\left(x_{0}\right)=\left\{x \in X: \omega_{\lambda}\left(x, x_{0}\right) \rightarrow 0 \text { as } \lambda \rightarrow \infty\right\}
$$


and

$$
X_{\omega}^{*}=X_{\omega}^{*}\left(x_{0}\right)=\left\{x \in X: \exists \lambda=\lambda(x)>0 \text { such that } \omega_{\lambda}\left(x, x_{0}\right)<\infty\right\}
$$

are said to be modular spaces (around $x_{0}$ ).

We obviously have $X_{\omega} \subset X_{\omega}^{*}$. In general this inclusion may be proper. It follows from $[9,10]$ that if $\omega$ is a modular on $X$, then the modular space $X_{\omega}$ can be equipped with a (nontrivial) metric, generated by $\omega$ and given by

$$
d_{\omega}(x, y)=\inf \left\{\lambda>0: \omega_{\lambda}(x, y) \leq \lambda\right\}
$$

for any $x, y \in X_{\omega}$. If $\omega$ is a convex modular on $X$, according to $[9,10]$ the two modular spaces coincide, i.e., $X_{\omega}^{*}=X_{\omega}$, and this common set can be endowed with the metric $d_{\omega}^{*}$ given by

$$
d_{\omega}^{*}(x, y)=\inf \left\{\lambda>0: \omega_{\lambda}(x, y) \leq 1\right\}
$$

for any $x, y \in X_{\omega}$. These distances will be called Luxemburg distances.

First attempts to generalize the classical function spaces of the Lebesgue type $L^{p}$ were made in the early 1930s by Orlicz and Birnbaum in connection with orthogonal expansions. Their approach consisted in considering spaces of functions with some growth properties different from the power type growth control provided by the $L^{p}$-norms. Namely, they considered the function spaces defined as follows:

$$
L^{\varphi}=\left\{f: \mathbb{R} \rightarrow \mathbb{R} ; \exists \lambda>0: \rho(\lambda f)=\int_{\mathbb{R}} \varphi(\lambda|f(x)|) d x<\infty\right\},
$$

where $\varphi:[0, \infty] \rightarrow[0, \infty]$ was assumed to be a convex function increasing to infinity, i.e., the function which to some extent behaves similarly to power functions $\varphi(t)=t^{p}$.

Modular function spaces $L^{\varphi}$ furnish a wonderful example of a modular metric space. Indeed define the function $\omega$ by

$$
\omega_{\lambda}(f, g)=\rho\left(\frac{f-g}{\lambda}\right)=\int_{\mathbb{R}} \varphi\left(\frac{|f(x)-g(x)|}{\lambda}\right) d x
$$

for all $\lambda>0$ and $f, g \in L^{\varphi}$, then $\omega$ is a modular metric on $L^{\varphi}$. Moreover, the distance $d_{\omega}^{*}$ is exactly the distance generated by the Luxemburg norm on $L^{\varphi}$.

For more examples on modular function spaces, the reader my consult the book of Kozlowski [11] and for modular metric spaces $[9,10]$.

Definition 4.3 Let $X_{\omega}$ be a modular metric space.

(1) The sequence $\left\{x_{n}\right\}_{n \in \mathbb{N}}$ in $X_{\omega}$ is said to be $\omega$-convergent to $x \in X_{\omega}$ if and only if $\omega_{1}\left(x_{n}, x\right) \rightarrow 0$ as $n \rightarrow \infty$. $x$ will be called the $\omega$-limit of $\left\{x_{n}\right\}$.

(2) The sequence $\left\{x_{n}\right\}_{n \in N}$ in $X_{\omega}$ is said to be $\omega$-Cauchy if $\omega_{1}\left(x_{m}, x_{n}\right) \rightarrow 0$ as $m, n \rightarrow \infty$.

(3) A subset $M$ of $X_{\omega}$ is said to be $\omega$-closed if the $\omega$-limit of a $\omega$-convergent sequence of $M$ always belongs to $M$.

(4) A subset $M$ of $X_{\omega}$ is said to be $\omega$-complete if any $\omega$-Cauchy sequence in $M$ is a $\omega$-convergent sequence and its $\omega$-limit is in $M$. 
(5) A subset $M$ of $X_{\omega}$ is said to be $\omega$-bounded if we have

$$
\delta_{\omega}(M)=\sup \left\{\omega_{1}(x, y) ; x, y \in M\right\}<\infty .
$$

(7) $\omega$ is said to satisfy the Fatou property if and only if for any sequence $\left\{x_{n}\right\}_{n \in \mathbb{N}}$ in $X_{\omega}$ $\omega$-convergent to $x$, we have

$$
\omega_{1}(x, y) \leq \liminf _{n \rightarrow \infty} \omega_{1}\left(x_{n}, y\right)
$$

for any $y \in X_{\omega}$.

In general if $\lim _{n \rightarrow \infty} \omega_{\lambda}\left(x_{n}, x\right)=0$ for some $\lambda>0$, then we may not have $\lim _{n \rightarrow \infty} \omega_{\lambda}\left(x_{n}\right.$, $x)=0$ for all $\lambda>0$. Therefore, as it is done in modular function spaces, we will say that $\omega$ satisfies $\Delta_{2}$-condition if this is the case, i.e., $\lim _{n \rightarrow \infty} \omega_{\lambda}\left(x_{n}, x\right)=0$ for some $\lambda>0$ implies $\lim _{n \rightarrow \infty} \omega_{\lambda}\left(x_{n}, x\right)=0$ for all $\lambda>0$. In [9] and [10], one will find a discussion about the connection between $\omega$-convergence and metric convergence with respect to the Luxemburg distances. In particular, we have

$$
\lim _{n \rightarrow \infty} d_{\omega}\left(x_{n}, x\right)=0 \quad \text { if and only if } \quad \lim _{n \rightarrow \infty} \omega_{\lambda}\left(x_{n}, x\right)=0 \quad \text { for all } \lambda>0
$$

for any $\left\{x_{n}\right\} \in X_{\omega}$ and $x \in X_{\omega}$. And in particular we have $\omega$-convergence and $d_{\omega}$ convergence are equivalent if and only if the modular $\omega$ satisfies the $\Delta_{2}$-condition. Moreover, if the modular $\omega$ is convex, then we know that $d_{\omega}^{*}$ and $d_{\omega}$ are equivalent, which implies

$$
\lim _{n \rightarrow \infty} d_{\omega}^{*}\left(x_{n}, x\right)=0 \quad \text { if and only if } \quad \lim _{n \rightarrow \infty} \omega_{\lambda}\left(x_{n}, x\right)=0 \quad \text { for all } \lambda>0
$$

for any $\left\{x_{n}\right\} \in X_{\omega}$ and $x \in X_{\omega}[9,10]$. Throughout this section, we assume that $\omega$ satisfies the Fatou property.

Let $(X, \omega)$ be a modular metric space and $G$ be a reflexive digraph defined on $X$.

Definition 4.4 Let $(X, \omega)$ and $G$ be as above. Let $C$ be a nonempty subset of $X$. The mapping $T: C \rightarrow C$ is said to be:

(i) G-monotone if $T$ is edge preserving, i.e., $(T(x), T(y)) \in E(G)$ whenever $(x, y) \in E(G)$ for any $x, y \in C$;

(ii) $G$-monotone $\omega$-quasi-contraction if $T$ is $G$-monotone and there exists $k<1$ such that for any $x, y \in C,(x, y) \in E(G)$, we have

$$
\begin{gathered}
\omega_{1}(T(x), T(y)) \leq k \max \left(\omega_{1}(x, y) ; \omega_{1}(x, T(x)) ; \omega_{1}(y, T(y)) ;\right. \\
\left.\omega_{1}(x, T(y)) ; \omega_{1}(y, T(x))\right) .
\end{gathered}
$$

Let $(X, \omega)$ be a modular metric space and $G$ be a reflexive digraph defined on $X$. We say that $E(G)$ has property $(* * *)$ if

$(* * *)$ For any $\left(x_{n}\right)_{n \geq 1}$ in $X$, if $x_{n} \omega$-converges to $x$ and $\left(x_{n}, x_{n+1}\right) \in E(G)$ for $n \geq 1$, then there is a subsequence $\left(x_{k_{n}}\right)_{n \geq 1}$ with $\left(x_{k_{n}}, x\right) \in E(G)$ for $n \geq 1$.

Note that if $G$ is a reflexive transitive digraph defined on $X$, then property (***) implies the following property: 
For any $\left(x_{n}\right)_{n \geq 1}$ in $X$, if $x_{n} \omega$-converges to $x$ and $\left(x_{n}, x_{n+1}\right) \in E(G)$ for $n \geq 1$, then $\left(x_{n}, x\right) \in E(G)$ for every $n \geq 1$.

Throughout this section, we assume that $(X, \omega)$ is a modular metric space, $G$ is a reflexive transitive digraph defined on $X$ and $E(G)$ has property $(* * *)$.

In the sequel we prove an analogue to Theorem 3.1 in modular metric spaces. For any $x \in C$, define the orbit $\mathcal{O}(x)=\left\{x, T(x), T^{2}(x), \ldots\right\}$, and its diameter by

$$
\delta_{\omega}(x)=\sup \left\{\omega_{1}\left(T^{n}(x), T^{m}(x)\right): n, m \in \mathbb{N}\right\}
$$

Throughout we assume that $\omega$ is regular and satisfies the Fatou property. The following technical lemma is crucial to prove the main result of this section. It is the modular version of Lemma 3.1. Its proof will be omitted.

Lemma 4.1 Let $(X, \omega)$ and $G$ be as above. Let $C$ be a nonempty subset of $X$ and $T: C \rightarrow C$ be a G-monotone $\omega$-quasi-contraction mapping. Let $x \in C$ be such that $(x, T(x)) \in E(G)$ and $\delta_{\omega}(x)<\infty$. Then, for any $n \geq 1$, we have

$$
\delta_{\omega}\left(T^{n}(x)\right) \leq k^{n} \delta_{\omega}(x)
$$

where $k<1$ is the constant associated with the G-monotone $\omega$-quasi-contraction definition of T. Moreover, we have

$$
\omega_{1}\left(T^{n}(x), T^{n+m}(x)\right) \leq k^{n} \delta_{\omega}(x)
$$

for any $n, m \in \mathbb{N}$.

Using Lemma 4.1, we prove the main result of this section.

Theorem 4.1 Let $(X, \omega)$ and $G$ be as above. Let $C$ be a nonempty subset of $X$ which is $\omega$-complete. Let $T: C \rightarrow C$ be a G-monotone $\omega$-quasi-contraction mapping. Let $x \in C$ be such that $(x, T(x)) \in E(G)$ and $\delta_{\omega}(x)<\infty$. Then:

(i) $\left\{T^{n}(x)\right\} \omega$-converges to $z \in C$ which is a fixed point of $T$ and $(x, z) \in E(G)$, provided $\omega_{1}(z, T(z))<\infty$ and $\omega_{1}(x, T(z))<\infty$. Moreover, we have

$$
\omega_{1}\left(T^{n}(x), z\right) \leq k^{n} \delta_{\omega}(x), \quad n \geq 1 .
$$

(ii) If $w$ is a fixed point of $T$ such that $(x, w) \in E(G)$ and $\omega_{1}\left(T^{n}(x), w\right)<\infty$ for any $n \geq 1$, then $z=w$.

Proof Let us prove (i). Lemma 4.1 implies that $\left\{T^{n}(x)\right\}$ is $\omega$-Cauchy. Since $C$ is $\omega$-complete, then there exists $z \in C$ such that $\left\{T^{n}(x)\right\} \omega$-converges to $z$. Since

$$
\omega_{1}\left(T^{n}(x), T^{n+m}(x)\right) \leq k^{n} \delta_{\omega}(x)
$$

for any $n, m \in \mathbb{N}$, the Fatou property (once we let $m \rightarrow \infty$ ) will imply

$$
\omega_{1}\left(T^{n}(x), z\right) \leq k^{n} \delta_{\omega}(x), \quad n \geq 1
$$


Since $T$ is $G$-monotone, we have $\left(T^{n}(x), T^{n+1}(x)\right) \in E(G)$ for any $n \geq 1$. Using property $(* * *)$, we get $\left(T^{n}(x), z\right) \in E(G)$ for any $n \geq \mathbb{N}$. In particular, we have $(x, z) \in E(G)$. Next we assume $\omega_{1}(z, T(z))<\infty$ and $\omega_{1}(x, T(z))<\infty$. Let us prove that $z$ is a fixed point of $T$. By induction, we have $\omega_{1}\left(T^{n}(x), T(z)\right)<\infty$, and

$$
\begin{aligned}
(\diamond) \quad \omega_{1}\left(T^{n}(x), T(z)\right) \leq & k \max \left(\omega_{1}\left(T^{n-1}(x), z\right) ; \omega_{1}\left(T^{n-1}(x), T^{n}(x)\right) ;\right. \\
& \left.\omega_{1}(T(z), z) ; \omega_{1}\left(T^{n-1}(x), T(z)\right) ; \omega_{1}\left(T^{n}(x), z\right)\right)
\end{aligned}
$$

for any $n \geq 1$. Consider $r(y)=\lim \sup _{n \rightarrow+\infty} \omega_{1}\left(T^{n}(x), y\right)$ for $y \in C$. From $(\diamond)$, we get

$$
\begin{aligned}
\omega_{1}\left(T^{n}(x), T(z)\right) & \leq k \max \left(k^{n-1} \delta_{\omega}(x) ; \omega_{1}(T(z), z) ; \omega_{1}\left(T^{n-1}(x), T(z)\right) ; k^{n} \delta_{\omega}(x)\right) \\
& =k \max \left(k^{n-1} \delta_{\omega}(x) ; \omega_{1}(T(z), z) ; \omega_{1}\left(T^{n-1}(x), T(z)\right)\right) \\
& \leq k^{n} \delta_{\omega}(x)+k \omega_{1}(T(z), z)+k \omega_{1}\left(T^{n-1}(x), T(z)\right) \\
& \leq \delta_{\omega}(x)+\omega_{1}(T(z), z)+k \omega_{1}\left(T^{n-1}(x), T(z)\right)
\end{aligned}
$$

for any $n \geq 1$. By induction, we obtain

$$
\omega_{1}\left(T^{n}(x), T(z)\right) \leq \frac{1}{1-k}\left(\delta_{\omega}(x)+\omega_{1}(T(z), z)\right)+k^{n} \omega_{1}(x, T(z))
$$

for any $n \geq 1$, which implies

$$
r(T(z)) \leq \frac{1}{1-k}\left(\delta_{\omega}(x)+\omega_{1}(T(z), z)\right)<+\infty .
$$

So if we let $n \rightarrow+\infty$ in the inequality

$$
\omega_{1}\left(T^{n}(x), T(z)\right) \leq k \max \left(k^{n-1} \delta_{\omega}(x) ; \omega_{1}(T(z), z) ; \omega_{1}\left(T^{n-1}(x), T(z)\right)\right)
$$

we get

$$
r(T(z)) \leq k \max \left(\omega_{1}(z, T(z)), r(T(z))\right) .
$$

Since $\omega$ satisfies the Fatou property, we get $\omega_{1}(z, T(z)) \leq r(T(z))$, which implies

$$
r(T(z)) \leq k \max \left(\omega_{1}(z, T(z)), r(T(z))\right)=k r(T(z)) .
$$

Since $k<1$, we conclude $r(T(z))=0$, which implies $\omega_{1}(z, T(z))=0$. Since $\omega$ is regular, we get $T(z)=z$.

Next we show (ii). Let $w \in C$ be a fixed point of $T$ such that $(x, w) \in E(G)$ and $\omega_{1}\left(T^{n}(x), w\right)<\infty$ for any $n \geq 1$. Then by induction we get

$$
\omega_{1}\left(T^{n}(x), w\right) \leq k \max \left(\omega_{1}\left(T^{n-1}(x), w\right) ; \omega_{1}\left(T^{n-1}(x), T^{n}(x)\right) ; \omega_{1}\left(T^{n}(x), w\right)\right)
$$

for any $n \geq 2$. Note that if

$$
\max \left(\omega_{1}\left(T^{n-1}(x), w\right) ; \omega_{1}\left(T^{n-1}(x), T^{n}(x)\right) ; \omega_{1}\left(T^{n}(x), w\right)\right)=\omega_{1}\left(T^{n}(x), w\right)
$$


for some $n \geq 1$, we get $\omega_{1}\left(T^{n}(x), w\right) \leq k \omega_{1}\left(T^{n}(x), w\right)$. Since $k<1$, we get $\omega_{1}\left(T^{n}(x), w\right)=0$. So $T^{n}(x)=w$, which implies $T^{n+m}(x)=w$ for any $m \geq 0$ since $w$ is a fixed point of $T$. This clearly will force $z=w$. Assume otherwise that

$$
\max \left(\omega_{1}\left(T^{n-1}(x), w\right) ; \omega_{1}\left(T^{n-1}(x), T^{n}(x)\right) ; \omega_{1}\left(T^{n}(x), w\right)\right) \neq \omega_{1}\left(T^{n}(x), w\right)
$$

for any $n \geq 2$. In this case, we get

$$
\omega_{1}\left(T^{n}(x), w\right) \leq k \max \left(\omega_{1}\left(T^{n-1}(x), w\right) ; k^{n-1} \delta_{\omega}(x)\right)
$$

for any $n \geq 2$. Hence

$$
\omega_{1}\left(T^{n}(x), w\right) \leq k \omega_{1}\left(T^{n-1}(x), w\right)+k^{n} \delta_{\omega}(x) \leq k \omega_{1}\left(T^{n-1}(x), w\right)+\delta_{\omega}(x)
$$

which implies by induction

$$
\omega_{1}\left(T^{n}(x), w\right) \leq k^{n} \omega_{1}(x, w)+\frac{1}{1-k} \delta_{\omega}(x)
$$

for any $n \geq 1$. In particular, we have $\limsup _{n \rightarrow \infty} \omega_{1}\left(T^{n}(x), w\right)<+\infty$. Using the inequality

$$
\omega_{1}\left(T^{n}(x), w\right) \leq k \max \left(\omega_{1}\left(T^{n-1}(x), w\right) ; k^{n-1} \delta_{\omega}(x)\right)
$$

for any $n \geq 2$, we obtain

$$
\limsup _{n \rightarrow \infty} \omega_{1}\left(T^{n}(x), w\right) \leq k \limsup _{n \rightarrow \infty} \omega_{1}\left(T^{n}(x), w\right)
$$

Since $k<1$, we get $\limsup _{n \rightarrow \infty} \omega_{1}\left(T^{n}(x), w\right)=0$, i.e., $\left\{T^{n}(x)\right\}$ converges to $w$. The uniqueness of the limit implies that $z=w$. Indeed, we have

$$
\omega_{2}(z, w) \leq \omega_{1}\left(T^{n}(x), z\right)+\omega_{1}\left(T^{n}(x), w\right), \quad n \geq 1
$$

If we let $n \rightarrow+\infty$, we get $\omega_{2}(z, w)=0$. Since $\omega$ is regular, we get $z=w$.

Note that under the assumptions of Theorem 4.1, if $w$ is another fixed point of $T$ such that $(w, z) \in E(G)$ and $\omega_{1}(z, w)<\infty$, then we have

$$
\omega_{1}(z, w)=\omega_{1}(T(z), T(w)) \leq k \omega_{1}(z, w)
$$

which implies $z=w$, since $k<1$.

The author declares that he has no competing interests.

\section{Acknowledgements}

The author acknowledges King Fahd University of Petroleum and Minerals for supporting this research.

Received: 13 February 2015 Accepted: 25 May 2015 Published online: 23 June 2015 
References

1. Banach, S: Sur les opérations dans les ensembles abstraits et leurs applications. Fundam. Math. 3, 133-181 (1922)

2. Nieto, JJ, Rodriguez-Lopez, R: Contractive mapping theorems in partially ordered sets and applications to ordinary differential equations. Order 22(3), 223-239 (2005)

3. Ran, ACM, Reurings, MCB: A fixed point theorem in partially ordered sets and some applications to matrix equations. Proc. Am. Math. Soc. 132(5), 1435-1443 (2004)

4. Jachymski, J: The contraction principle for mappings on a metric space with a graph. Proc. Am. Math. Soc. 136, 1359-1373 (2008)

5. Khamsi, MA, Kirk, WA: An Introduction to Metric Spaces and Fixed Point Theory. Wiley, New York (2001)

6. Bachar, M, Khamsi, MA: On monotone Ćirić quasi-contraction mappings. Preprint

7. Vetro, C, Vetro, F: Metric or partial metric spaces endowed with a finite number of graphs: a tool to obtain fixed point results. Topol. Appl. 164, 125-137 (2014)

8. Cirić, LB: A generalization of Banach's contraction principle. Proc. Am. Math. Soc. 45, 267-273 (1974)

9. Chistyakov, W: Modular metric spaces, I: basic concepts. Nonlinear Anal. 72(1), 1-14 (2010)

10. Chistyakov, W: Modular metric spaces, Il: application to superposition operators. Nonlinear Anal. 72(1), 15-30 (2010)

11. Kozlowski, WM: Modular Function Spaces. Series of Monographs and Textbooks in Pure and Applied Mathematics, vol. 122. Dekker, New York (1988)

\section{Submit your manuscript to a SpringerOpen ${ }^{\circ}$ journal and benefit from:}

- Convenient online submission

- Rigorous peer review

Immediate publication on acceptance

- Open access: articles freely available online

- High visibility within the field

- Retaining the copyright to your article 